\title{
Ultrashort-Pulse Light-Assisted Periodic Poling of Lithium Niobate and Lithium Tantalate
}

\author{
C. E. Valdivia, C. L. Sones, S. Mailis, R. W. Eason \\ Optoelectronics Research Centre, University of Southampton, Southampton SOI 7 I BJ, United Kingdom
}

$c$

\begin{abstract}
We report the light-assisted poling of controllable periodic and arbitrarily-shaped domain patterns in lithium niobate and lithium tantalate via the simultaneous application of a low uniform DC electric-field and sequence of ultrashort pulses (150-200 fs).
\end{abstract}

\section{Introduction}

The lithium niobate (LN) and lithium tantalate (LT) family of crystals are of interest for their large optical nonlinearity and electro-optic coefficients, and are therefore used in applications such as quasi-phase-matched (QPM) parametric generation and modulators. Nonlinear interactions are achieved in these materials by periodically inverting the spontaneous polarization every coherence length in a procedure called electric-field poling. By applying an electric-field in excess of the coercive field, $E_{\mathrm{c}} \sim 22 \mathrm{kV} / \mathrm{mm}$, switching between the two anti-parallel domain states can be achieved for photolithographically-defined periods down to several microns. However, shorter periods are required for applications such as near-ultraviolet generation, backward-propagating parametric frequency conversion, and electro-optic Bragg gratings.

The application of light provides a further dimension of control for the formation of ferroelectric domains that may permit the advanced functionality enabled by shorter periods while removing the need for lithographic processing. All-optical poling using only ultraviolet (UV) nanosecond-pulsed light with a fluence around the ablation threshold has been shown to form self-organized nano-scale domain patterns on the $+z$ face of lithium niobate [1]. Alternatively, light-assisted poling (LAP) is a process whereby a uniform DC electric field biases a crystal during patterned illumination for the formation of inverted domain structures in the illuminated regions only. Using this technique, domains have been formed using electric-fields as low as $50 \%$ below $E_{\mathrm{c}}$ using continuouswave (cw) UV light from a mercury lamp [2] and an $\mathrm{Ar}^{+}$laser [3] illuminating $\mathrm{MgO}$-doped congruently melting lithium niobate (Mg:CLN). Visible light has also been used to directly-write domains with electric-fields $30 \%$ below $E_{\mathrm{c}}$ in undoped CLN [4], and up to $90 \%$ below $E_{\mathrm{c}}$ in $\mathrm{Mg}: \mathrm{CLN}$ and MgO-doped near-stoichiometric LN (Mg:SLN) when doped above their optical damage thresholds [5].

In this paper, we describe the poling conditions and results for ultrashort-pulsed UV, visible, and near-infrared (NIR) light-assisted poling in lithium niobate and lithium tantalate crystals.

\section{Procedure}

The setup for LAP consisted of a transparent cell holding LN or LT crystals, with uniform water electrodes on either face. Each crystal first underwent five "dark" electric-field poling cycles (without illumination), inverting and reinverting a uniform area. This process is observed using a CCD camera, with domain boundaries revealed through stress-induced birefringence and electro-optic refractive index contrast. The $-z$ face of the crystal was then illuminated by an ultrashort-pulsed laser system while simultaneously applying a uniform DC electric-field. An optical parametric amplifier (OPA) supplied pulse durations of 150-200 fs with wavelengths tunable from NIR to UV regions. The materials investigated include undoped CLN, Mg:CLN doped above the optical damage threshold, and undoped congruently melting lithium tantalate (CLT). All experiments were conducted at room temperature Further details of the setup and procedure are provided in Ref [5].

\section{Results}

The purpose of these experiments is to investigate the light-induced modification of the poling behavior, in particular, at the microscopic scale. The dark nucleation field, $E^{\text {nucl }}(I=0)$, is defined as the lowest electric-field for which microscopic domain nucleation is observed in the absence of illumination, and is slightly below the 
conventionally-defined coercive field. In general, the nucleation field, $E^{\text {nucl }}(I)$, is the lowest electric-field for which nucleation occurs under an illumination of intensity, $I$.

The poling behavior using ultrashort-pulse LAP is strongly dependent upon the parameters of electric field, light intensity, and exposure time (number of pulses). Conceptually, this may be viewed as similar to pulsed electric-field poling, where a sequence of millisecond-duration pulses first nucleates then spreads a domain, and a comparison with pulsed-LAP will be reported.

Ultrashort-pulse LAP has been exploited to produce both surface and bulk domains using wavelengths $\lambda=800$, $514.5,400,383,364,334,305 \mathrm{~nm}$. Both visible and UV wavelengths have been observed to reduce the minimum nucleation field by up to $98 \%$ in $\mathrm{Mg}: \mathrm{CLN}$, producing a further 5-fold advantage over $\mathrm{cw}$ experiments [5]. This reduction permits domain inversion with the use of electric fields as low as $100 \mathrm{~V} / \mathrm{mm}$. Directly-written domain patterns exhibit arbitrarily-shaped structures strongly dependent upon the shape of the beam. This includes directlywritten bulk domains having walls that are not confined to the crystalline symmetry, and can follow circular and arbitrarily-angled lines on both the $+z$ and $-z$ faces. Illuminating the crystal through a phase mask has also been used to periodically pole Mg:CLN with a period $\Lambda=5.25 \mu \mathrm{m}$, as seen in Figure 1. Scanning the beam over a static phase mask and sample can potentially increase the length of the QPM grating for efficient device applications, while maintaining optimal exposure conditions without the need to expand the beam size.
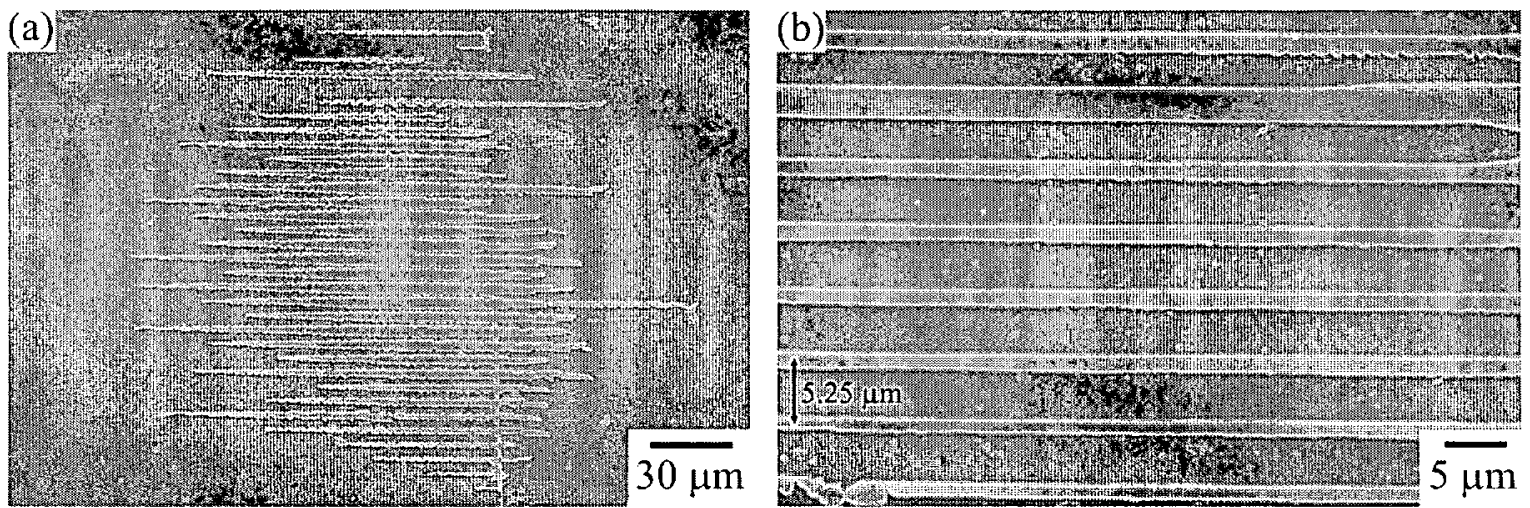

Figure 1: Light-assisted poling of $5-\mathrm{mol} \% \mathrm{Mg}$-doped CLN with ultrashort-pulsed light of $\lambda=514.5 \mathrm{~nm}$, using (a) static exposure of a Gaussian beam with bias of $E=1200 \mathrm{~V} / \mathrm{mm}$ and $I_{\mathrm{av}} \sim 2 \mathrm{~W} / \mathrm{cm}^{2}$, and (b) scanned exposure with a bias of $E=800 \mathrm{~V} / \mathrm{mm}$ and $I_{\mathrm{av}} \sim 20 \mathrm{~W} / \mathrm{cm}^{2}$.

Undoped congruent lithium tantalate has also permitted light-assisted poling of inverted domain structures below its coercive field using ultrashort-pulsed light of $\lambda=400 \mathrm{~nm}$, exhibiting a similar reduction of the nucleation field as observed in undoped CLN. Directly-written domain structures are possible, confined closely to the lateral extent of the beam. To the author's knowledge, this is the first time light has been shown to reduce the nucleation field in LT. Further investigations of this effect are in progress.

\section{Conclusion}

Illumination with ultrashort-pulsed light introduces another degree of control over domain engineering, removing the need for photolithography and permitting the use of low voltages, with a further 5-fold reduction compared to cw light in Mg:CLN. Periodic domain patterning has been achieved using interference methods, producing useful domain sizes of approximately the micron scale. Future experiments will endeavor to achieve functional devices with shorter periods in both lithium niobate and lithium tantalate crystals.

\section{References}

[1] C.E. Valdivia, C.L. Sones, J.G. Scott, S. Mailis, R.W. Eason, D.A. Scrymgeour, V. Gopalan, T. Jungk, E. Soergel, and I. Clark, "Nanoscale surface domain formation on the $+z$ face of lithium niobate by pulsed ultraviolet laser illumination," Appl. Phys. Lett., 86, 022906 (2005).

[2] M. Fujimura, T. Sohmura, and T. Suhara, "Fabrication of domain-inverted gratings in $\mathrm{MgO}: \mathrm{LiNbO}_{3}$ by applying voltage under ultraviolet irradiation through photomask at room temperature," Electron. Lett., 39, 719-21 (2003).

[3] M.C. Wengler, U. Heinemeyer, E. Soergel, and K. Buse, "Ultraviolet light-assisted domain inversion in magnesium-doped lithium niobate crystals," J. Appl. Phys., 98, 064104 (2005).

[4] V. Dierolf and C. Sandmann, "Direct-Write Method for Domain Inversion Patterns in LiNbO,," Appl. Phys. Lett., 84, 3987-9 (2004).

[5] C.L. Sones, M.C. Wengler, C.E. Valdivia, S. Mailis, R.W. Eason, and K. Buse, "Light-induced order-of-magnitude decrease in the electric field for domain nucleation in MgO-doped lithium niobate crystals," Appl. Phys. Lett., 86, 212901 (2005). 